\title{
Primary care for diabetes mellitus: perspective from older patients
}

\author{
This article was published in the following Dove Press journal: \\ Patient Preference and Adherence \\ 6 October 2011 \\ Number of times this article has been viewed
}

\author{
Eliza Lai Yi Wong' \\ Jean Woo ${ }^{2}$ \\ Elsie $\mathrm{Hui}^{3}$ \\ Carrie Chan ${ }^{2}$ \\ Wayne LS Chan ${ }^{2}$ \\ Annie Wai Ling Cheung' \\ 'School of Public Health and Primary \\ Care, The Chinese University of \\ Hong Kong, ${ }^{2} \mathrm{School}$ of Public Health \\ and Primary Care, Division of \\ Geriatrics, Department of Medicine \\ and Therapeutics, Faculty of Medicine, \\ The Chinese University of Hong \\ Kong, ${ }^{3}$ Medical and Geriatric Unit, \\ Shatin Hospital, HK SAR, Hong Kong, \\ People's Republic of China
}

Background: Care of diabetes mellitus in the elderly requires an additional perspective to take into account impaired cognitive function, physical function, low level of education, and difficulty making lifestyle changes. Existing services tend to be driven by the views of tertiary and secondary care staff, rather than those of primary care staff and elderly patients. This study aimed to explore the attitudes and preferences of elderly patients with diabetes mellitus towards primary care (clinical care and community program).

Method: Elderly patients with diabetes mellitus aged 60 years or above were recruited from governmental diabetes mellitus clinics and diabetes mellitus specific community centers. Three focus group discussions of 14 diabetic elderly patients were conducted and their perspectives on the new service model were assessed. Participants were interviewed according to an open-ended discussion guide which includes the following items: comments on existing clinic follow up and community program, motivation for joining the community program, and suggestions on further clinical services and community service program development.

Results: Incapability of the current health service to address their special needs was a common concern in three focus group discussions. The majority highlighted the benefits of the new service program, that is, self-care knowledge and skill, attitudes to living with diabetes mellitus, and supportive network. Key facilitators included experiential learning, a group discussion platform, and goal setting with patients.

Conclusions: This study is the first qualitative study to explore the views of elderly diabetic patients' on their self-care needs. Elderly people with diabetes mellitus in this study identified bad experiences of clinical follow-up; benefit from the community program; and recommendations for the future development of primary care. Study findings revealed a number of discrepancies between elderly diabetic patients' needs and existing health services in Hong Kong. The study findings provide health practitioners, researchers and educators with an additional perspective on the provision of quality of care for elderly diabetic patients in the community.

Keywords: primary care, diabetes, self-care

\section{Introduction}

Type 2 diabetes mellitus affects about $85 \%-95 \%$ of people with diabetes in developed countries and the percentage is even higher in developing countries. ${ }^{1}$ McGhee et al showed that the prevalence of diabetes rises with age, and estimated that $50 \%$ of patients with diabetes mellitus in Hong Kong are aged 65 years or older. ${ }^{2}$ Type 2 diabetes is therefore a growing problem in the older population, resulting in a significant burden for the individual, families, and society. ${ }^{3}$ There is evidence that better self-management can improve the health outcomes of patients with diabetes. ${ }^{4,5}$ Self-management is defined as patients taking an active part in the management of
Correspondence: Eliza Lai Yi Wong 2/F, School of Public Health Building, Prince of Wales Hospital, Shatin, New Territories, Hong Kong, People's Republic of China Tel +85222528772

Fax +85226063500

Email lywong@cuhk.edu.hk 
their chronic conditions with appropriate clinical, social, and emotional support. The American Diabetes Association recommends self-management of diabetes including dietary adjustment, physical activity, management of diabetes medications, and compliance with medical follow-up for monitoring, as an integral component of all patient care plans. ${ }^{6}$ Thus, it is important to establish a self-management approach for patients with diabetes in primary care. However, several studies have shown that deteriorating functional and cognitive ability hinders their self-care management ${ }^{7-10}$ and increases the use of both health and social services. ${ }^{11}$ Cognitive and functional impairment are common complications in patients with diabetes, ${ }^{12-14}$ this disability is particularly apparent in the elderly. ${ }^{15,16}$ Hendra and Sinclair showed that the primary care management approach for older patients differs from that for younger adults because of the presence of age-related changes in functional and mental ability. ${ }^{17}$

The views of patients are an important basis for developing self-care management. A study conducted in adults with diabetes mellitus indicated that empowerment in selfmanagement would make them more positive. ${ }^{18}$ Studies identified certain factors that hindered self-management, ie, poor physical condition/physical immobility; financial constraints; transport problems; long waiting times at clinics; unsupportive healthcare professionals; and lack of home blood glucose monitoring device, family support, and long-term educational/psychological intervention. ${ }^{18-20}$ The needs of elderly people with diabetes differ from those of younger people, because of their greater vulnerability as a result of functional and cognitive impairment. ${ }^{15,16}$ However, these particular needs of elderly patients have received little attention to date.

Care of diabetes mellitus in older persons requires an additional perspective to take into account impaired cognitive function, physical function and low education level among the elderly, and difficulty in making lifestyle changes. The clinic follow-up services in Hong Kong (out-patient clinics on medical consultation and treatment) and nurse-led clinics (follow-up on treatment compliance, screening for foot and eye problems, and reinforcing self-management skills in blood glucose checking, diet and exercise) do not take these particular needs into account. Clinic services tend to be driven by tertiary and secondary care staff perspectives rather than by primary care and elderly patients' perspectives. Many different types of community programs are being launched in Hong Kong. Their domains mainly focus on exercise prescription, diabetes care education and self-management techniques (on treatment and monitoring compliance). The format of the existing community program could be regular/irregular, intensive/on-off and long-term/short-time, etc. The attitudes underlying both clinic follow-up and community-based programs for the self-management of elderly patients with diabetes are unknown. The findings of this study could provide important insights for researchers, educators, policy makers, and healthcare professionals, to develop primary care for community-dwelling elderly with diabetes, locally and internationally.

\section{Aims and objectives}

The aim of the study was to reveal the perceptions of community-dwelling elderly with diabetes on the role of existing primary care (clinic follow-up and community-based program) in self-management. The study aimed: (1) To investigate the attitudes towards the clinic follow-up and community-based program for elderly with diabetes; and (2) To explore suggestions for the future development of primary care services to enhance self-management in elderly with diabetes.

\section{Methods Design and sample}

A qualitative descriptive study using a focus group discussion was conducted with participants joining the new communitybased diabetes mellitus program in Hong Kong. Three focus group discussions comprising 4-6 patients with type 2 diabetes mellitus in each group were conducted at community elderly centers. The patients were consecutively recruited from governmental specialist clinics (diabetes mellitus) and diabetes mellitus specific community centers using convenience sampling. All participants were aged 60 years or over, Chinese, spoke Cantonese, and able to give verbal consent. There were no sex limitations to study entry. However, participants who were unable to provide informed consent or who were deaf or mute were not included. The participants' recruitment continued until issues were felt to be data saturated and no new relevant data seemed to emerge.

\section{Measures}

The focus group discussion was led by two experienced researchers (C Chan and WLS Chan) using semi-structured open-ended questions, derived from an in-depth literature review, and reviewed by the research team. The questions were divided into three topics: (1) attitudes towards the role of existing primary care services (clinic follow-up and community-based program) on the self-management of elderly people with diabetes; (2) motivation to join the 
community-based program; (3) suggestions for further development of primary care (clinic care and a communitybased program). The focus group discussions were conducted at the elderly community centers, at times convenient for the individuals participating in the study. The discussions lasted up to 60 minutes and were audio-recorded with the participants' agreement. To increase study validity, all focus group discussions were conducted by the same facilitator (C Chan). Demographics of the participants, including age, sex, educational attainment, medical history of diabetes mellitus, and exercise time per week were also collected.

\section{Analytical strategy}

All focus group discussions were audio-recorded and transcribed by one of the team members (C Chan). Content analysis was undertaken and themes identified independently by two members of the team (ELY Wong and C Chan). The content analysis was used for data analysis because it placed an emphasis on the conceptual meaning of the role of primary care in participants' own words and expression, which was our aim in this study. ${ }^{21}$ Data analysis and focus group discussion were concurrently conducted to check the data saturation. Themes identified from coding were agreed in team meetings, by discussion and consensus, on attitudes toward primary care for diabetes mellitus, motivation to join the community programs, and suggestions on the future development of primary care for diabetes mellitus from the perspective of community-dwelling elderly people with diabetes mellitus.

\section{Ethical considerations}

Ethical approval for the study was obtained from the university ethics committee. All potential participants were informed of the purpose of the study, the right to study participation and to withdrawal from the study at any time. Verbal consent was then obtained from all participants prior to focus group discussion.

\section{Results}

\section{Characteristics of participants}

Three focus groups were held between January and March 2008 in Hong Kong. Data collection and analysis were conducted concurrently, and recruitment ceased when data saturation was reached. A total of 14 participants were included in the discussions and their ages ranged from 61 to 94 (mean $=69.79 ; \mathrm{SD}=8.248)$. Most of them were male $(9 / 14=64.3 \%)$, had secondary school or higher educational attainment $(9 / 14=64.3 \%)$, participated regularly in some sort of physical activity $(9 / 14=64.3 \%)$, had coexisting hypertension $(10 / 14=71.4 \%)$ and/or other chronic diseases with medication, and had previously been admitted to hospital as a result of diabetes mellitus $(10 / 14=71.4 \%)$. Their demographics are shown in Table 1.

The central conclusion of this study was that participants acknowledged that the management of diabetes entails selfresponsibility. Most participants perceived the majority of community programs as beneficial for enhancing selfmanagement in respect of maintaining physical activity and healthy diet, awareness of the importance of controlling a stable blood glucose level, and reinforcement of a positive attitude towards living with diabetes mellitus. It was important for patients to feel that long-term engagement with the program is the key to maintaining day-to-day selfcare. Patients appreciated the supportive nursing care in the existing clinical service, but most of them felt negatively

Table I Demographics of participants $(n=14)$

\begin{tabular}{|c|c|c|}
\hline & Mean (SD) & n (\%) \\
\hline Age & $69.79(8.248)$ & \\
\hline \multicolumn{3}{|l|}{ Sex } \\
\hline Male & & $9(64.3)$ \\
\hline Female & & $5(35.7)$ \\
\hline \multicolumn{3}{|l|}{ Education level } \\
\hline None & & I (7.I) \\
\hline No formal education & & I (7.I) \\
\hline Primary & & $3(21.4)$ \\
\hline Secondary or higher & & $9(64.3)$ \\
\hline Duration of diabetes mellitus (years) & $15.50(8.364)$ & \\
\hline \multicolumn{3}{|l|}{ Number of other chronic diseases } \\
\hline Hypertension & & $10(71.4)$ \\
\hline Chronic lung disease & & $3(21.4)$ \\
\hline Hyperlipidemia & & $6(42.9)$ \\
\hline Heart disease & & $2(14.3)$ \\
\hline Stroke & & $2(14.3)$ \\
\hline Osteoarthitis & & I (7.I) \\
\hline Hepatitis & & $3(21.4)$ \\
\hline Renal disease & & $\mathrm{I}(7.1)$ \\
\hline \multicolumn{3}{|l|}{ Exercise } \\
\hline None & & $3(2 \mathrm{I} .4)$ \\
\hline Occasionally & & $2(14.3)$ \\
\hline Regularly & & $9(64.3)$ \\
\hline Days of exercise per week & 4.71 (2.998) & \\
\hline \multicolumn{3}{|l|}{ Type of exercises } \\
\hline None & & $3(2 I .4)$ \\
\hline Walking & & $8(57.1)$ \\
\hline Tai Chi & & $\mathrm{I}(7.1)$ \\
\hline Others & & $2(14.3)$ \\
\hline $\begin{array}{l}\text { Previous admission to hospital due } \\
\text { to diabetes mellitus }\end{array}$ & & $10(71.4)$ \\
\hline \multicolumn{3}{|l|}{ Number of medications } \\
\hline $1-5$ & & $5(35.7)$ \\
\hline $6-10$ & & $6(42.9)$ \\
\hline $11-15$ & & $3(21.4)$ \\
\hline
\end{tabular}


about the doctors' unsympathetic attitudes, long waiting times for consultation and concern that their needs might not be addressed. In all, four themes were identified in the focus group discussions: (1) comments on the existing clinical service, which included attending out-patient and nurse-led clinics to receive information about the course of the illness, care, and rehabilitation, and to discuss medical treatment; (2) experiences of participation in the community programs; (3) motivation to join the new service model; (4) suggestions for the further development of the new service model.

\section{Attitudes towards clinical follow-up}

Most participants knew the importance of going to the clinic, and but they described several negative experiences including long waiting times, unhelpful consultation sessions, and poor relationships with doctors. However, the participants appreciated the nursing care in screening for complications.

\section{Long waiting times}

Most participants highlighted the long waiting times for consultations and medication dispensing at the clinic. The following comments were typical:

We have to wait for a long time for consultation ... the consultation and drug dispensing usually take me 6 to 7 hours, almost half of the day.

\section{Unhelpful consultations}

Most participants expressed the view that consultation times were insufficient for them to discuss problems related to controlling their diabetes such as increased immobility and dependency:

They (the doctors) are impatient to listen to you ... maybe their workload is very heavy ... we don't have a chance to tell him the detailed medical situation ... he just asks how the situation is and how the medication compliance is ... not more than two questions ....

\section{Professionals' attitudes}

The participants described a lack of support from the doctors. The patients felt that the doctors were uncompassionate and impatient because they always focused on the documentation throughout the consultation. The following comments typify responses provided in this area:

He (the doctor) is always typing the computer and writing notes .... you (the patient) complain of some discomfort but he (the doctor) does not even glance at you ... just says $\mathrm{OK} \ldots$ and then prints out the prescription and asks you to leave ... only 5 minutes' consultation ... no chance to ask questions.

The patients, however, felt the nurses were more considerate and supportive because they showed more concern and were more approachable during consultation. The following comments typify responses provided in this area:

The nurse is much better than before ... they are very kind and approachable.

The nurse makes a detailed check on my situation for 5 hours including ECG, eye check-up, urine sugar checking, and preparation of appointment slips for the next follow-up.

\section{Attitudes towards community programs}

The findings suggest that patients appreciated and benefited from the lifestyle modification class on self-management. They gained competence, knowledge, and skills to strengthen self-management. The availability and content of the lifestyle modification class were perceived as providing important support for patients' self-management in the following respects.

\section{Increase diabetes-related knowledge and skills}

The participants expressed a wide range of impressions about healthy lifestyle, including knowledge and skills on diet, exercise, foot care, and blood glucose. Most participants consequently changed their self-care behavior with a view to controlling their diabetes mellitus. Typical comments are summarized in Table 2.

\section{Enhance positive attitudes towards diabetes-related self-management}

Patients expressed themselves as happier and with more positive mind-sets about living with diabetes mellitus. The following comment identifies this perception:

For emotional health, I will not think any unhappy thoughts ... more psychologically relaxed ....

\section{Build a supportive network}

Feedback from patients revealed that emotional support and assistance in self-management were gained from the class. The following comment was typical:

I'm so happy to know so many friends ... We can remind each other about building a healthy habit ... so we will do more exercise, which will promote health. 
Table 2 Typical comments in view of the benefit from the program

\begin{tabular}{|c|c|}
\hline Aspects & Comments \\
\hline \multirow[t]{6}{*}{ Overall } & "I have changed ... I can do more exercise and eat more carefully ... because the life (health) is mine. \\
\hline & "My lifestyle becomes more structured ..." \\
\hline & "The length of each session is appropriate ... the contents are very informative and the progresses \\
\hline & of teaching contents are fine to me" \\
\hline & "I especially like the small group discussion instead of large group ... I like to exercise with my \\
\hline & friends together here instead of home because we can remind each other." \\
\hline \multirow[t]{2}{*}{ Diabetes-related knowledge } & "I know more about my situation (diabetes mellitus) ... I have gained a lot by each topic \\
\hline & (lifestyle modification topic) ...” \\
\hline \multirow[t]{2}{*}{ Blood glucose level } & "It do help me to stabilize my blood glucose level ..." \\
\hline & "I am more aware of my blood glucose level now." \\
\hline Medical treatment & "I know how to take the medication (correctly)" \\
\hline \multirow[t]{2}{*}{ Diet } & "It help me to remember what food I should avoid to eat ... and I know the right portion \\
\hline & of each food" \\
\hline \multirow[t]{4}{*}{ Exercise } & "I have learnt how to perform exercise ... skipping rope ... walking ... pulling rubber band/rope ... \\
\hline & and some aerobic exercise ... so I have more physical activity now" \\
\hline & "I have performed more exercise than before ... I know the exercise habit must be long-lasting ... \\
\hline & must insist on this principle" \\
\hline Foot care & "I can take care of myself ... trimming foot nails, choosing right shoes ... foot care is importance \\
\hline & to prevent complications (of diabetes mellitus)" \\
\hline
\end{tabular}

\section{Motivation to join community program} Gain knowledge and skills to facilitate self-management of diabetes mellitus

Participants expressed a wide range of positive impressions about the class in their appreciation of its format on availability, content, and style which fitted their needs. The following comments were made:

I want to learn more to control my diabetes mellitus ... I would like some continuous reminder in order to have a correct and positive belief (on the management of diabetes mellitus).

\section{Suggestions for the further development of the new service model}

Most participants desired to have a long-term program as a continuous reminder and reinforcement of the proper self-management of diabetes mellitus. They would also like more outdoor/in-class activity and seminar leaflets that could reinforce self-management skills.

At my age, the memory is not good, so I would like to join this program to remind us about the importance of exercise, diet ... otherwise, I will forget or find it hard in the long term to adhere to what we have learned ... so, I want to have a long-term program on a weekly basis, so the nurse can check and remind me about my exercise pattern, diet, ... so then I can remember the rules.

It would be good to have more activity, eg, one activity (outdoor or in-class) every 8 classes ... the message will be more impressive.
The participants suggested that the program be promoted to patients who were newly diagnosed or were young, with a view to diminishing complications as early as possible.

Recruitment in the elderly home may not be good because there are patients with long histories of diabetes mellitus and they may not be interested in following the rules or any changes in lifestyle any more ... they are more stubborn. The program should start to recruit those with newly diagnosed diabetes mellitus ... or youngsters ... for example, at the health clinic. They are more concerned and willing to change their lifestyles.

Concern about emotional health and stress management was prominent in the participants' accounts. The participants viewed the management of diabetes mellitus as a cause of stress, which further worsens the symptoms. Some participants talked about relieving stress with the comfort of an uncontrolled diet. They expressed the view that proper stress management should be an important component of the program.

Living with diabetes mellitus is very stressful and so I always forget the rules ... then lose control of eating and drinking ... in turn the diabetes mellitus becomes more serious. Sometime, I enjoy releasing stress with drinking beer or red wine with friends ... one cup after another ... or one box after another ... non-stop ... then I can fall asleep without any pressure. So, it would be helpful to know how to relieve stress properly. 


\section{Discussion}

This qualitative study provides insights about the attitudes of elderly patients towards the role of the primary care services of diabetes self-management. All study participants were aware of the importance of self-management in their diabetic care and held the opinion that the existing clinical service (clinically initiated session) was incapable of meeting their needs, while highlighting encouraging feedback about the community-based program. These findings confirm previous reports on the special needs of elderly diabetic patients. ${ }^{17,22}$

The patients appreciated the care they received from nursing staff, but most believed that the existing service had limited capacity to facilitate patient's self-management in their diabetic care such as lack of self-care skills teaching, insufficient emotional support, and inadequate access to medical follow-up (long waiting time). The special demands of elderly diabetic patients were mentioned by Sinclair and Barnett who identified that coexisting and often chronic medical problems, such as cerebrovascular disease, arthritis, Parkinson's disease and dementia, in the elderly exacerbate the impact of diabetes, increase the level of disability, and hinder management. ${ }^{22} \mathrm{~A}$ diabetic clinic may therefore not meet the needs of the diabetic elderly because of time constraints of healthcare professionals in relation to high workload. In addition to reporting an inadequate amount of discussion about prognosis, the manner of doctors and the quality of care were also described by the participants. They felt neglected during consultations, which had a routine structure and did not ascertain individual patients' preferred information requirements. Participants' preoccupation with these feelings might mean that they did not assimilate the information and advice given. Indeed a high rate of non-adherence to the treatment of diabetes mellitus in the older population has been perceived by clinicians. ${ }^{23}$ On the other hand, patients were left with undisclosed concerns and also felt that the information given had been inadequate for their needs. Similar lines of research have highlighted the need for clear and caring delivery of health care information..$^{24,25}$ Boulton reported that poor follow-up might mean inadequate screening for complications, failure to provide sufficient information and education to both patients and carers, and an increased rate of hospital admission as a result. ${ }^{26}$ The focus of clinical evaluation should be on the illness experiences of the patient, rather than the disease alone so that the meaning of the illness for the patient may be understood and realistic treatment goals established. Patient-directed documentation would increase the understanding of patients' concerns and disease progress by health care professionals, and patients would thus receive timely advice and care, in order to decrease avoidable hospitalization. Harris showed that a more structured interview emphasizes rational thought and focuses on gathering factual information; an effective clinical encounter should elicit attitudes, feelings and facts. ${ }^{27}$

A number of positive feedback comments about the community-based program emerged: increasing diabetesrelated knowledge and skill for maintaining a healthy lifestyle; enhancing positive attitudes towards diabetes-related self-management; and building a supportive network. The community-based program in small groups was conducive to increased self-engagement in diabetic care, as was evident in the patients' reports. Crucially, the regular and intensive community-based program could engender a positive attitude in the patients to their condition; this is one of the important primary guidelines for developing an education program for elderly suggested by Sinclair et al. ${ }^{28}$ Self-management skills including problem solving within the peer group incorporated into the educational program could enhance the experiential learning of diabetic self-care among elderly patients. Also, most of the participants said they enjoyed the guided group exercise very much. This experiential learning, rather than instructions and information during individual counselling on lifestyle changes, could promote independence, confidence, and self-sufficiency among elderly patients by taking account of their greater difficulty in self-management as a result of impaired cognitive function, physical function, and low educational level. ${ }^{17} \mathrm{We}$ feel that incorporating the evaluation of short-term and achievable self-management goals set by the patients is an essential starting point for developing self-management skills. Any model of care for managing the elderly diabetic patient should recognize the important role of the individual patient in educating, assessing, and goalsetting, as well as the development of shared care protocols. These findings highlight an important way of engaging the elderly patient in diabetic care, which considers their special needs, attitudes, and fears. . $^{17,19,22}$

The participants suggested that the community-based program should be a long-term sustainable program for information refreshment and updating. Although it is common and simple knowledge, effective information recall is important for elderly people with significant and increasing cognitive dysfunction. A number of studies have reported similar findings. ${ }^{18,23}$ In addition, frequent contact in the longterm program could provide an effective means of screening the elderly patient's disability level in order to tailor an appropriate self-management model to their needs. Also, 
most of them suggested incorporating stress management skills into the program. This concern was also highlighted in another study showing that poor psychological functioning causes more suffering, which can seriously interfere with daily diabetes self-management and is associated with poor medical outcomes and high costs. ${ }^{29}$ To achieve better diabetes care, we should not only focus on the acute and chronic complications of diabetes mellitus but also improve patients' psychological well-being and quality of life. ${ }^{30}$ Some participants further suggested that the community-based program should be presented at an early stage of diabetes mellitus in order to avoid the development of incorrect concepts and habits. An educational program during the early stage with timely involvement of specialist care and appropriate support to carers will be more beneficial for self-management in diabetic care.

This study has several limitations. The comments on primary care service including the clinic follow-up and community-based program were based on the perspective of patients, patients' family/carer as a potential facilitator or barrier was not acknowledged by the participants in the context of self-management. This discrepancy provides a potential area for further study in diverse family situations. The baseline level of functional ability and cognitive ability was not included; the history of diabetes mellitus and co-morbidity were used as a proxy of patients' health conditions. Our results may reflect participation bias, because the sample was recruited from the governmental specialist clinic and diabetes specific community program, and the perspectives of elderly people who missed follow-up by the clinic or who did not join the community program were not included. Future study to include both groups of participants (active and non-active) is important.

\section{Conclusion}

This qualitative study provides unique insights into the attitudes and comments of elderly patients about the discrepancy between the existing health services and their needs. The positive feedback about the community-based program may add to the literature on strategies for addressing the special needs of the elderly, when considering impairment of cognitive and physical function. The findings from this study also identify a number of suggestions for future development of community-based programs including a sustainable and longterm program on a regular and intensive basis, stress management skills, and commencement of diabetes self-management at an early stage of the disease. This study will help the development of primary care aimed at promoting self-management among the elderly - an important component of the healthcare service to improve care outcomes in this population.

\section{Disclosure}

The authors declare that they have no competing interests in this work.

\section{References}

1. International Diabetes Federation. Diabetes Atlas. 3rd ed. Brussels, Belgium: International Diabetes Federation; 2008.

2. McGhee SM, Cheung WL, Woo J, et al. Trends of Disease Burden Consequent to Diabetes in Older Persons in Hong Kong: Implications of population ageing: Hong Kong Jockey Club Charities Foundation (in press); 2009.

3. Dunstan DW, Zimmet PZ, Welborn TA, et al. The rising prevalence of diabetes and impaired glucose tolerance: the Australian Diabetes, Obesity and Lifestyle Study. Diabetes Care. 2002;25(5):829-834.

4. The Diabetes Control and Complications Trial Research Group. The effect of intensive treatment of diabetes on the development and progression of long-term complications in insulin-dependent diabetes mellitus. N Engl J Med. 1993;329(14):977-986.

5. Williams GC, McGregor HA, Zeldman A, Freedman ZR, Deci EL. Testing a self-determination theory process model for promoting glycemic control through diabetes self-management. Health Psychol. 2004;23(1):58-66.

6. American Diabetes Association. Standards of medical care in diabetes 2009. Diabetes Care. 2009;32(Suppl 1):S13-S61.

7. Ciechanowski PS, Katon WJ, Russo JE, Hirsch IB. The relationship of depressive symptoms to symptom reporting, self-care and glucose control in diabetes. Gen Hosp Psychiatry. 2003;25(4):246-252.

8. Maty SC, Fried LP, Volpato S, Williamson J, Brancati FL, Blaum CS. Patterns of disability related to diabetes mellitus in older women. J Gerontol A Biol Sci Med Sci. 2004;59(2):148-153.

9. Park H, Hong Y, Lee H, Ha E, Sung Y. Individuals with type 2 diabetes and depressive symptoms exhibited lower adherence with self-care. J Clin Epidemiol. 2004;57(9):978-984.

10. Sinclair AJ, Bayer AJ, Girling AJ, Woodhouse KW. Older adults, diabetes mellitus and visual acuity: a community-based case-control study. Age Ageing. 2000;29(4):335-339.

11. Sinclair AJ, Girling AJ, Bayer AJ. Cognitive dysfunction in older subjects with diabetes mellitus: impact on diabetes self-management and use of care services. All Wales Research into Elderly (AWARE) Study. Diabetes Res Clin Pract. 2000;50(3):203-212.

12. Kalmijn S, Feskens EJ, Launer LJ, Stijnen T, Kromhout D. Glucose intolerance, hyperinsulinaemia and cognitive function in a general population of elderly men. Diabetologia. 1995;38(9):1096-1102.

13. Vanhanen M, Koivisto K, Kuusisto J, et al. Cognitive function in an elderly population with persistent impaired glucose tolerance. Diabetes Care. 1998;21(3):398-402.

14. Sinclair AJ, Conroy SP, Bayer AJ. Impact of diabetes on physical function in older people. Diabetes Care. 2008;31(2):233-235.

15. Boult C, Kane RL, Louis TA, Boult L, McCaffrey D. Chronic conditions that lead to functional limitation in the elderly. $J$ Gerontol. 1994;49(1):M28-M36.

16. Gregg EW, Beckles GL, Williamson DF, et al. Diabetes and physical disability among older US adults. Diabetes Care. 2000;23(9):1272-1277.

17. Hendra TJ, Sinclair AJ. Improving the care of elderly diabetic patients: the final report of the St Vincent Joint Task Force for Diabetes. Age Ageing. 1997;26(1):3-6.

18. Wong M, Haswell-Elkins M, Tamwoy E, McDermott R, d'Abbs P. Perspectives on clinic attendance, medication and foot-care among people with diabetes in the Torres Strait Islands and Northern Peninsula Area. Aust J Rural Health. 2005;13(3):172-177. 
19. Carbone ET, Rosal MC, Torres MI, Goins KV, Bermudez OI. Diabetes self-management: perspectives of Latino patients and their health care providers. Patient Educ Couns. 2007;66(2):202-210.

20. Wellard SJ, Rennie S, King R. Perceptions of people with Type 2 diabetes about self-management and the efficacy of community based services. Contemp Nurse. 2008;29(2):218-226.

21. Catherine P, Nicholas M. Qualitative Research in Health Care (3rd ed). Oxford, UK: Blackwell Publishing; 2006.

22. Sinclair AJ, Barnett AH. Special needs of elderly diabetic patients. BMJ. 1993;306(6886):1142-1143.

23. Forbes A, Berry J, While A, Hitman G, Sinclair AJ. Issues and methodological challenges in developing and evaluating health care interventions for older people with diabetes mellitus, part 1. Pract Diab Int. 2002;19(2):55-59.

24. McGrath P. End-of-life care for hematological malignancies: the 'technological imperative' and palliative care. J Palliat Care. 2002; 18(1):39-47.
25. Kirk P, Kirk I, Kristjanson LJ. What do patients receiving palliative care for cancer and their families want to be told? A Canadian and Australian qualitative study. BMJ. 2004;328(7452):1343.

26. Boulton AJ. Update on long-term diabetic complications. In: Lewin IG, Seymour CA, editors. Current Themes in Diabetic Care. London: Royal College of Physicians of London; 1992.

27. Harris JC. Toward a restorative medicine - the science of care. JAMA. 2009;301(16):1710-1712.

28. Sinclair AJ, Turnbull CJ, Croxson SC. Document of care for older people with diabetes. Special Interest Group in Diabetes, British Geriatrics Society. Postgrad Med J. 1996;72(848):334-338.

29. Egede LE, Zheng D, Simpson K. Comorbid depression is associated with increased health care use and expenditures in individuals with diabetes. Diabetes Care. 2002;25(3):464-470.

30. Debono M, Cachia E. The impact of diabetes on psychological well being and quality of life. The role of patient education. Psychol Health Med. 2007;12(5):545-555.

\section{Publish your work in this journal}

Patient Preference and Adherence is an international, peer-reviewed, open access journal focusing on the growing importance of patient preference and adherence throughout the therapeutic continuum. Patient satisfaction, acceptability, quality of life, compliance, persistence and their role in developing new therapeutic modalities and compounds to optimize clinical outcomes for existing disease states are major areas of interest. This journal has been accepted for indexing on PubMed Central. The manuscript management system is completely online and includes a very quick and fair peer-review system. Visit http://www.dovepress.com/ testimonials.php to read real quotes from published authors. 